\title{
Visualization of Kallikreins in Thyroid Epithelial and Carcinoma Cells
}

Eileen Kroll, Anastasija Pejkovska, Maren Rehders, Adam Lesner, Grzegorz Dubin, Tomasz Kantyka, Dagmar Führer, Jan Potempa, Klaudia Brix

\section{INTRODUCTION}

Kallikrein expression in thyroid tissue ${ }_{\text {trat }}$

Molecular structure of tissue kallikreins (KLK) KLK2 - KLK3/PSA - KLK4 - KLK5 - KLK7 - KLK11 - KLK12 - KLK13 - KLK14 - KLK15

Kallikreins (KLKs) are afamily ofhormonally regulated proteinases comprising 15 related enzzymes that are expressed in many tissues and celltypes. The aim of this study is KLian insights into synchronization studies were conducted toinvestigate de novo biosynthesis and transport pathways ofKLKs during cell cycle progression.

$\mathrm{N}-$ Pre Pro Serine-protease domain - C

TSH stimulation of Nthy-ori 3-1 cells triggers secretion of kallikreins like KLK2 and KLK3/PSA

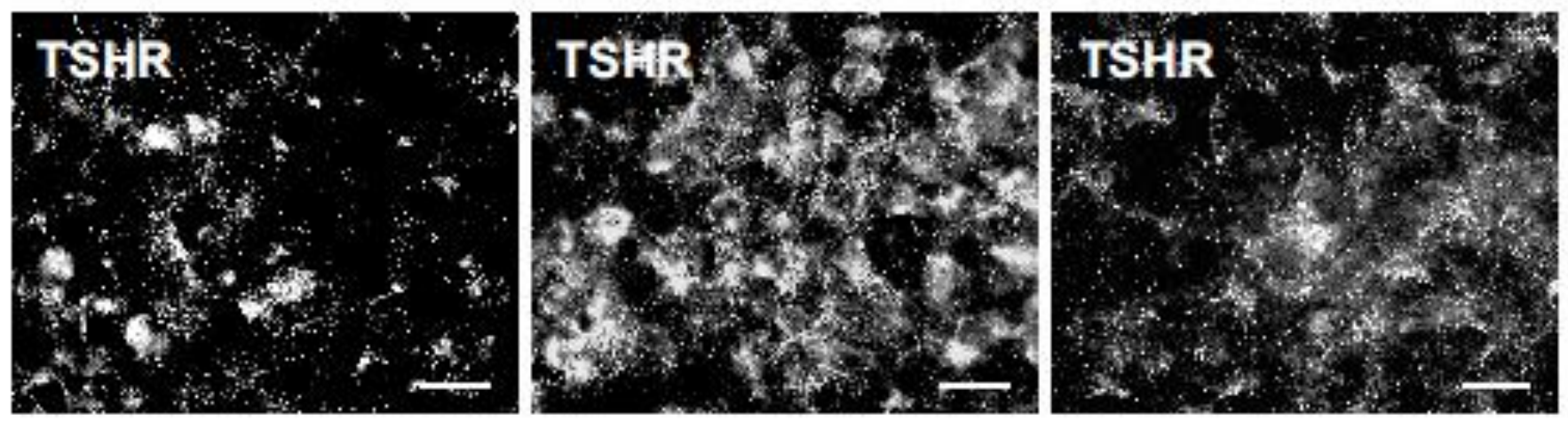

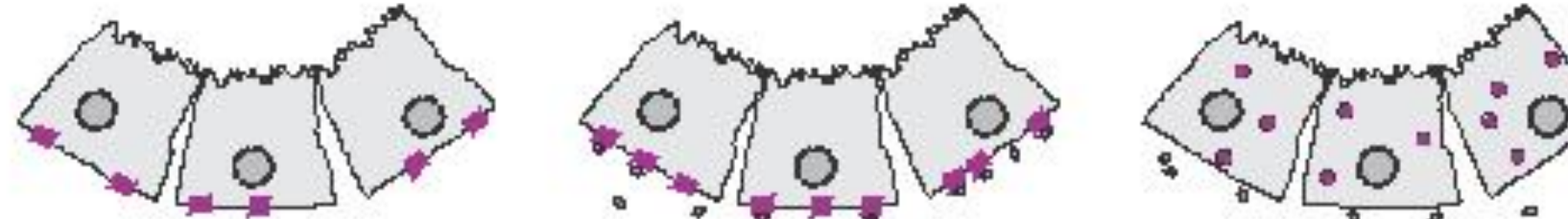

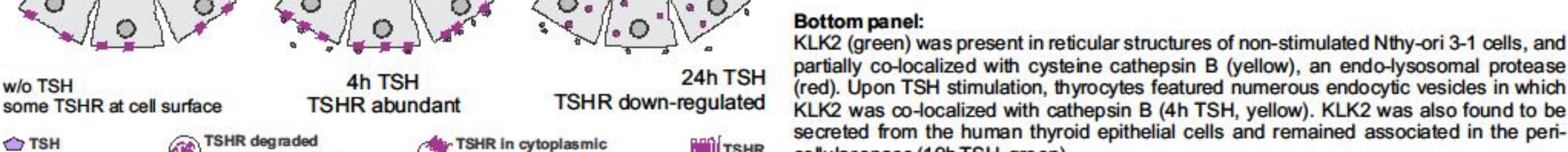
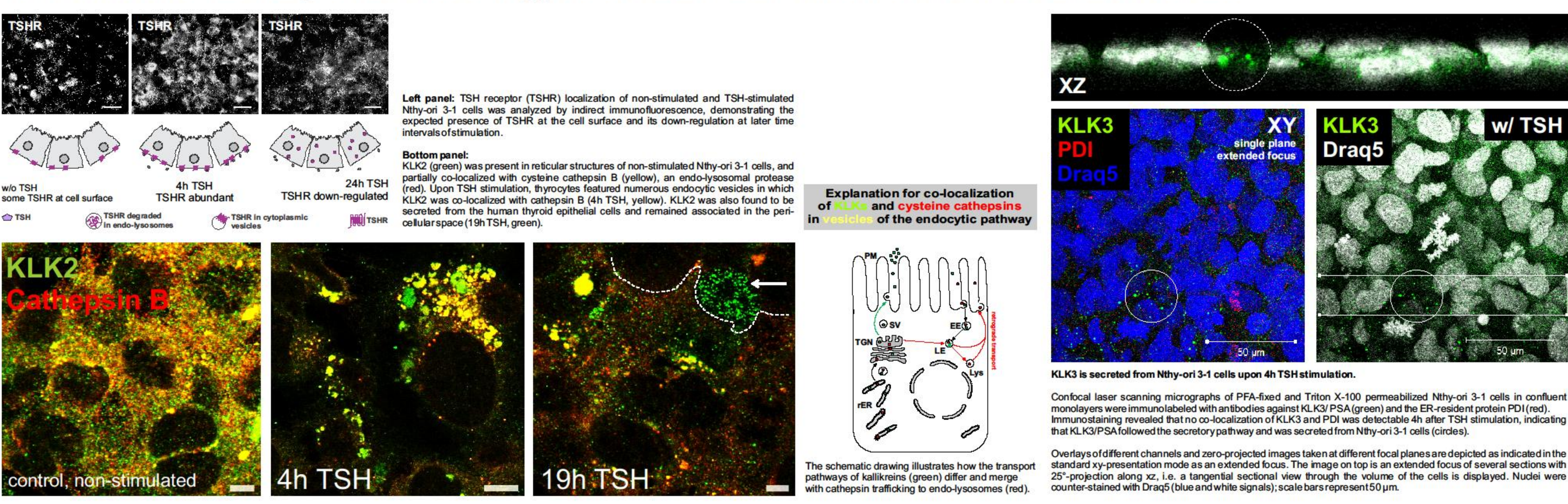

Synchronization in G1-phase and subsequent TSH stimulation results in up-regulated de novo biosynthesis of KLK3

TSH-stimulation during release from cell cycle arrest in G1/G0-phase

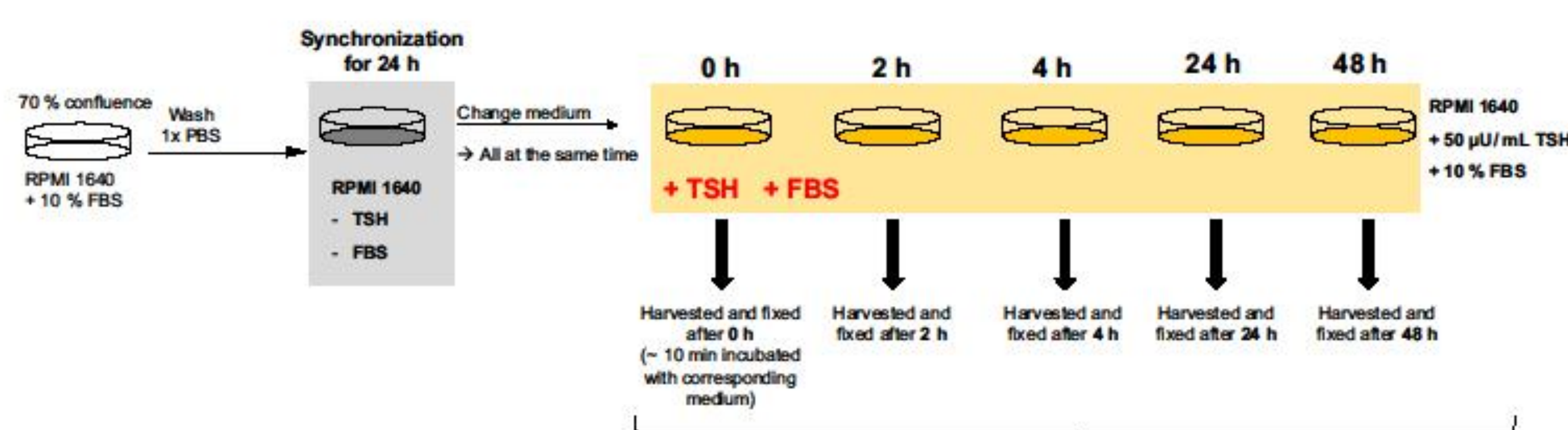

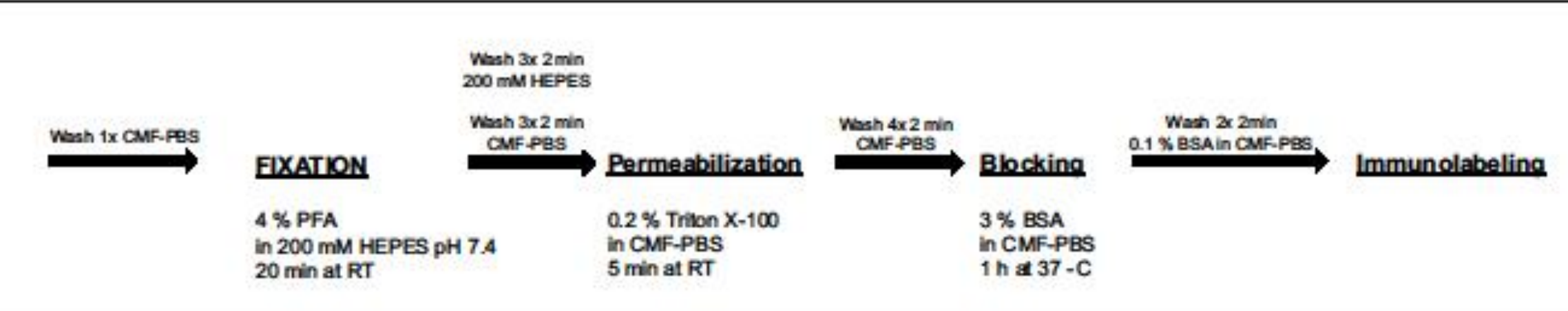

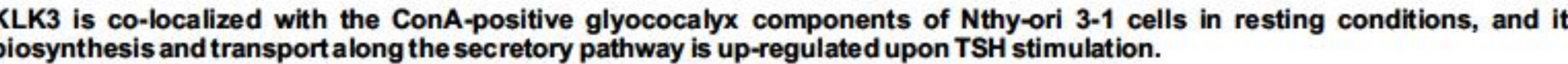

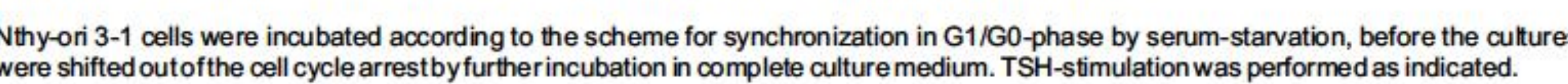

Thyroid cell lines to analyze cancer states in vitro

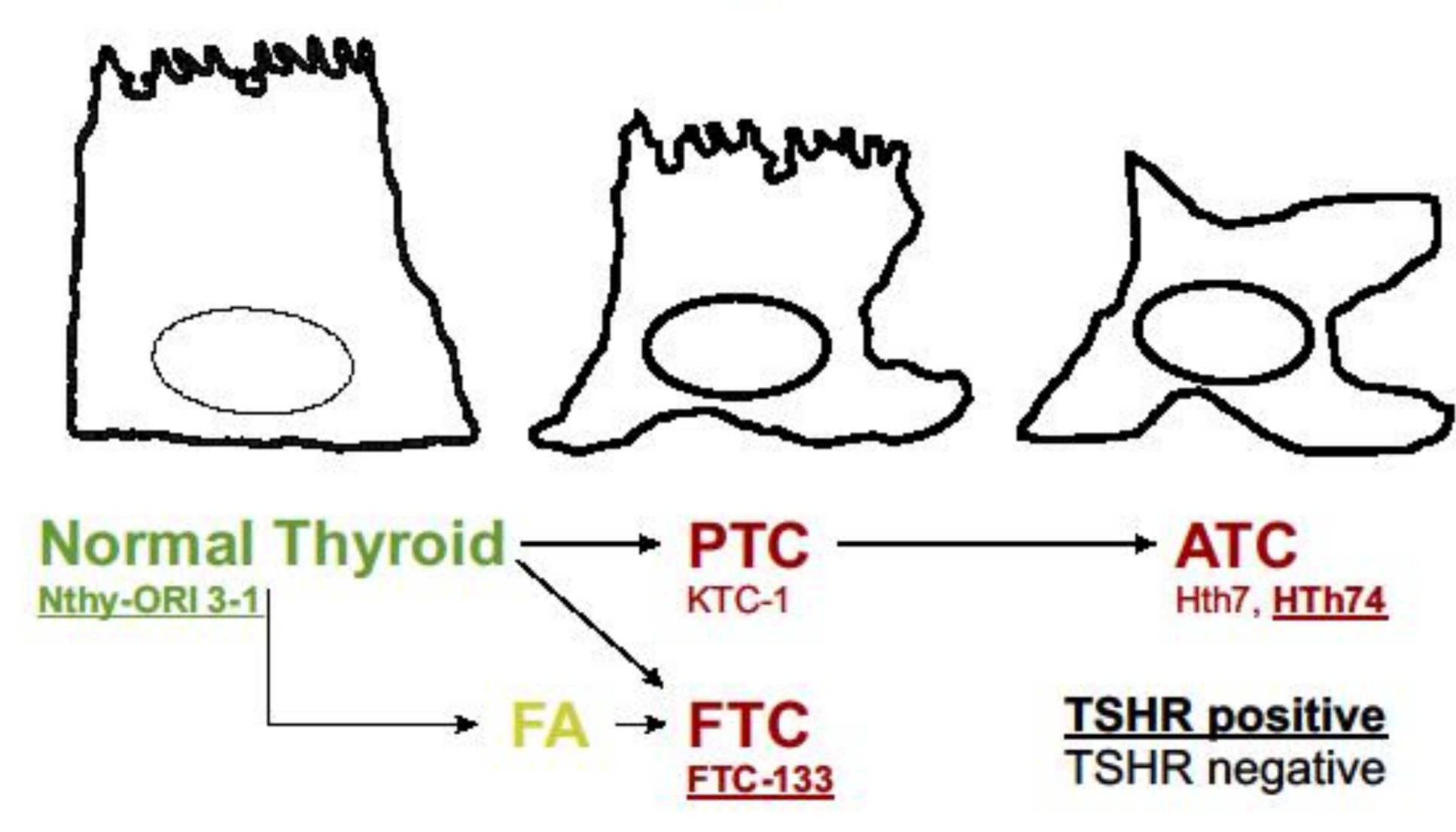

KTC-1
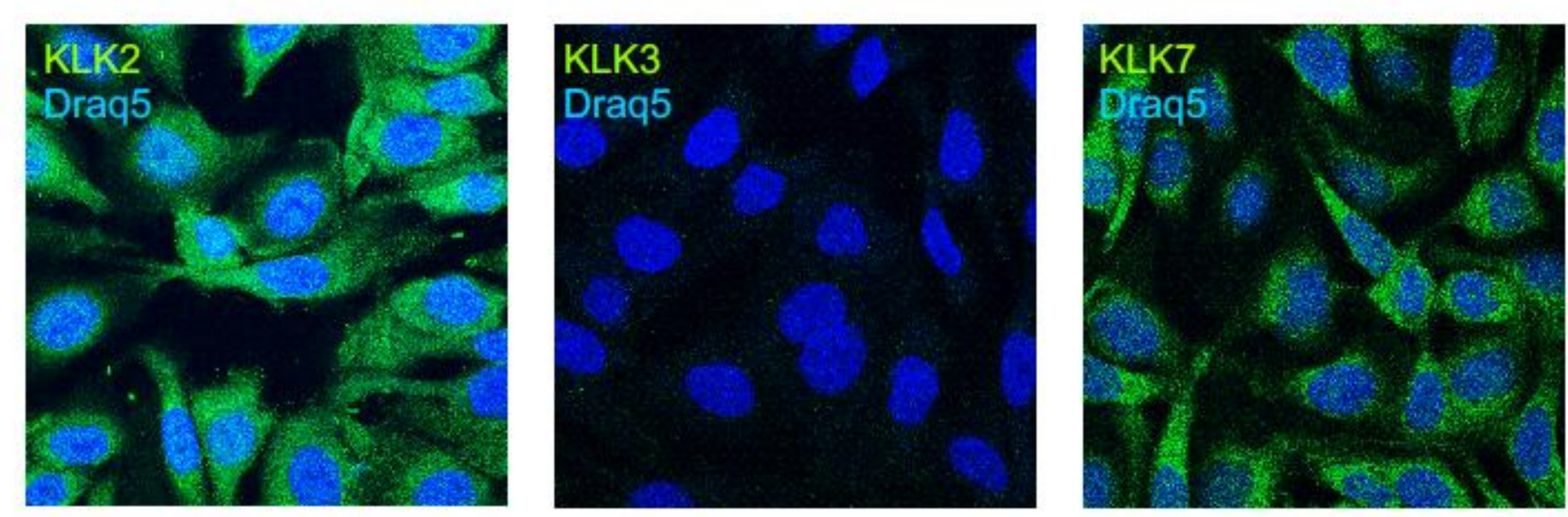

FTC-133
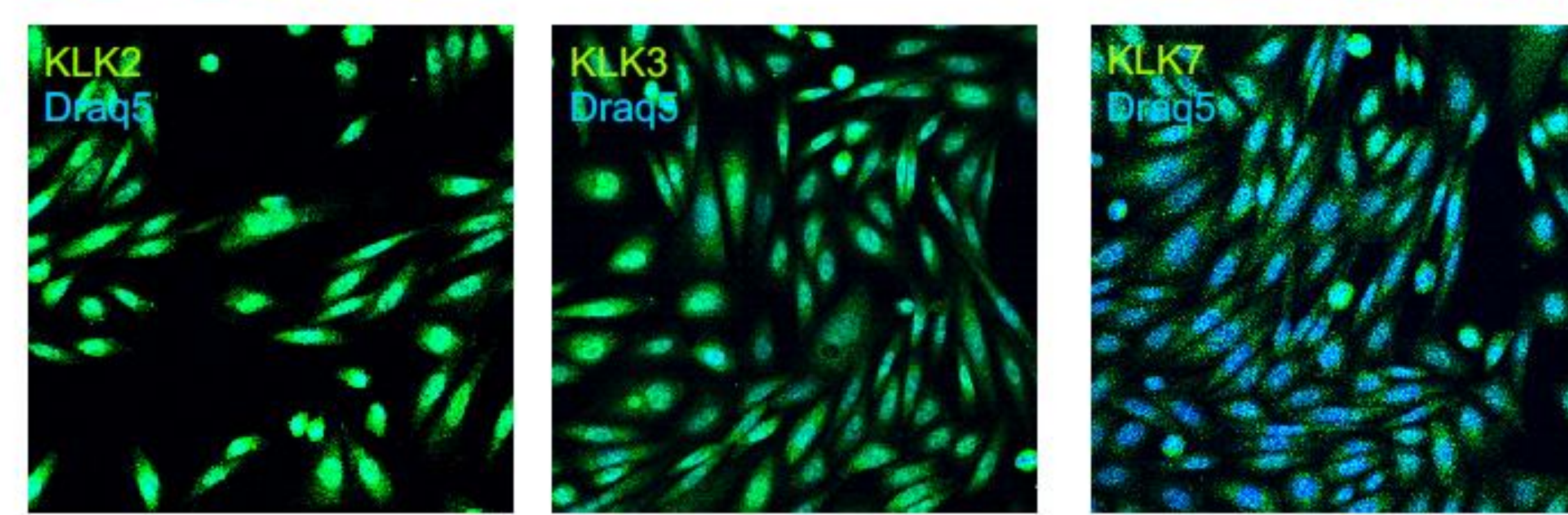

KLK2, KLKK3/PSA, and KLKT
prevalentin particularin in $F$.

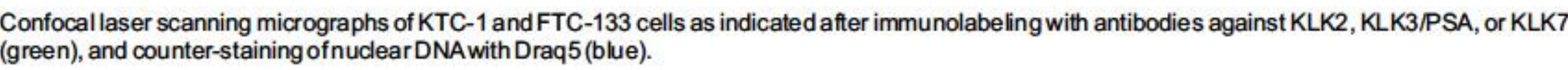

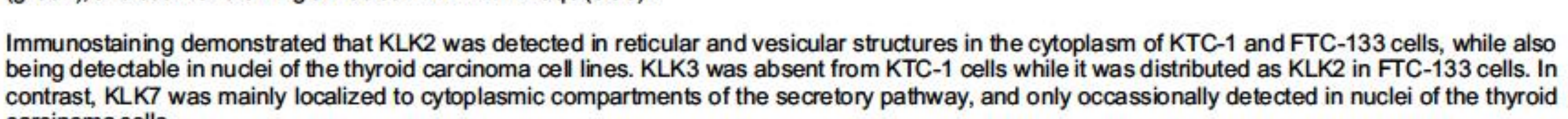

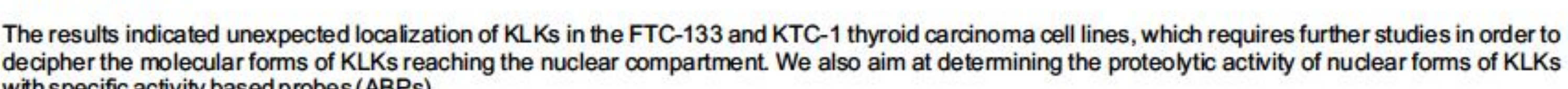
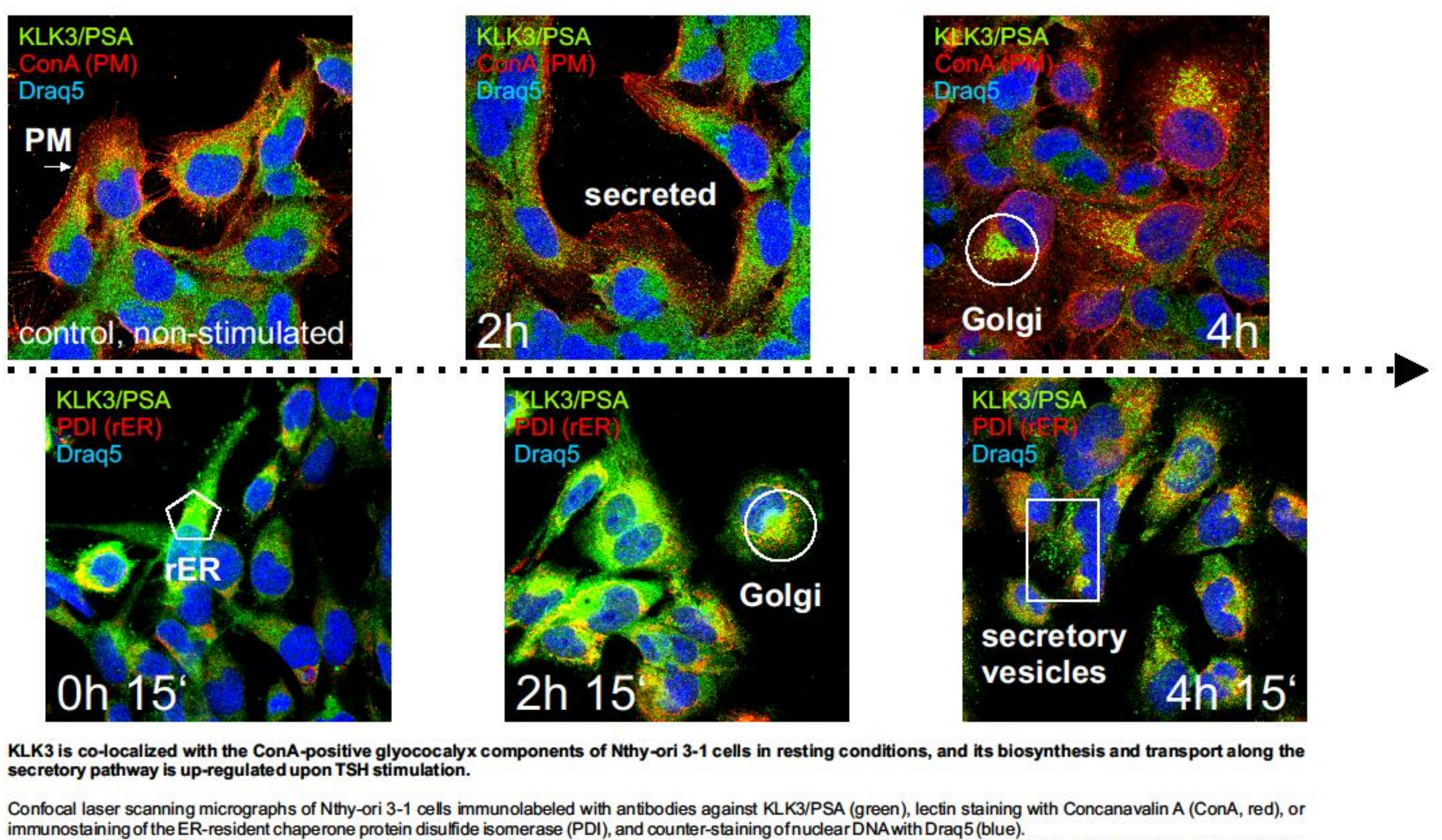

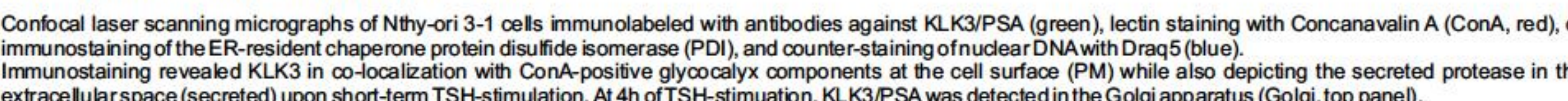

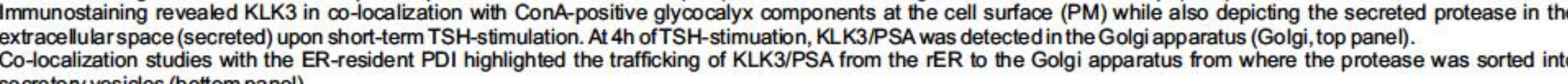

\section{CONCLUSION}
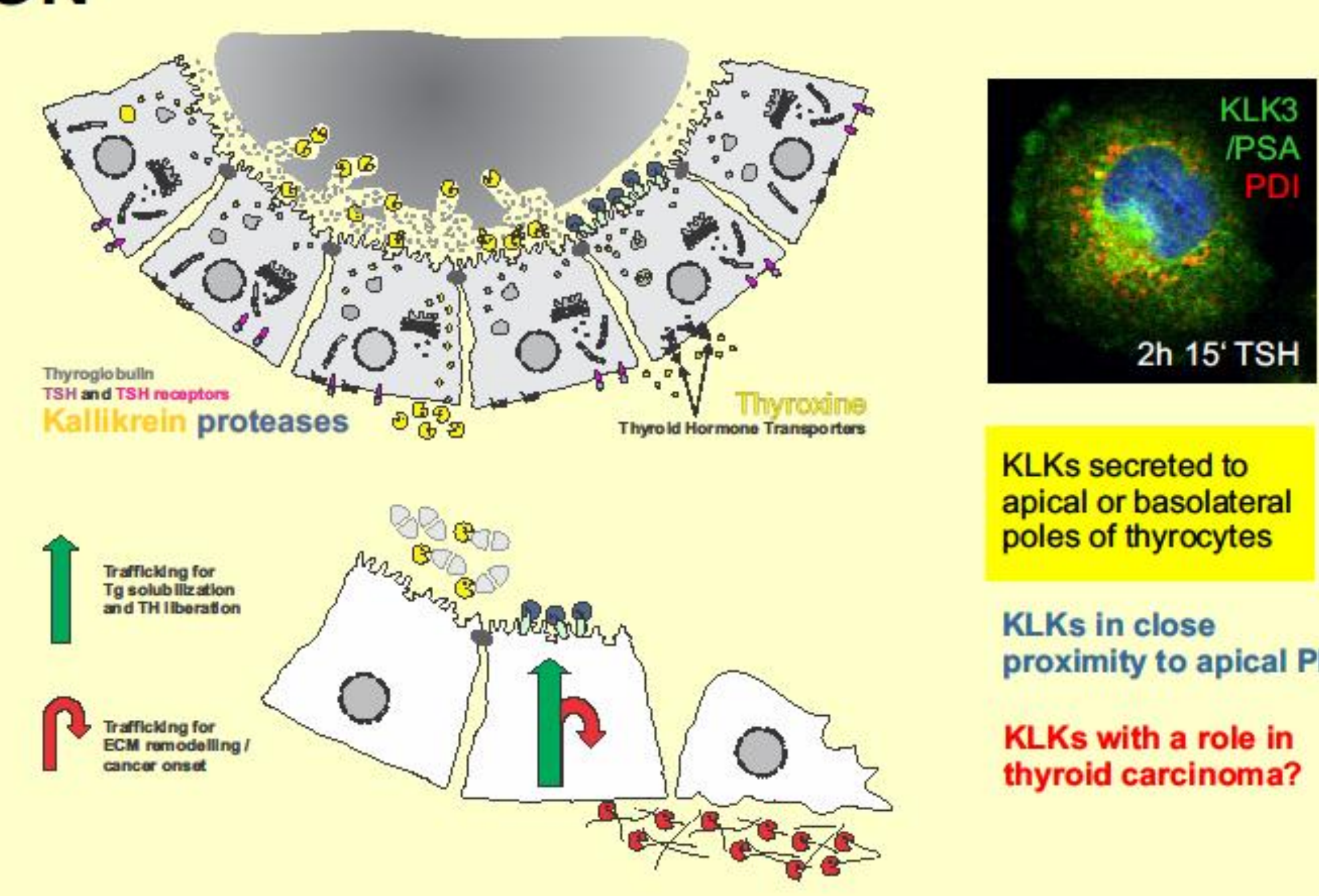

KLKs in close
proximity to apical PM

KLKs with a role in
thyroid carcinoma?

Schematic representation of the observed localization of KLKs in thyroid epithelial anc carcinoma cells In addition to their presence in compartments of the secretory and endocytic pathways, KLKs were

The presence of KLKs in extracellular compartments of thyroid tissue might indicate their secretion at the and thyroid hormone liberation from the precursor molecule, which was so far demonstrated for thyroid cysteine cathepsins [Friedrichs et al., 2003; Jordans et al., 2009; Dauth et al., 2011].

Basolateral secretion of KLKs (red arrow) proposes that they might also be involved in ECM degration thus contributing to cancer onset and progression. A novel function of KLKs is envisioned for specific

in the nuclei of thyroid carcinoma cells (pink symbols) [Brix et al 2015]

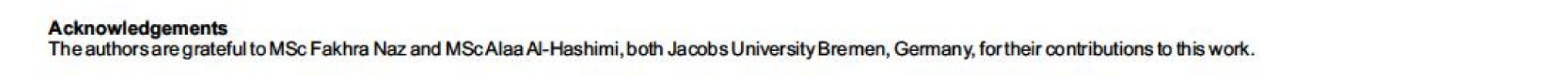

